\title{
Why children absorb more microwave radiation than adults
}

\author{
L. Lloyd Morgan
}

Environmental Health Trust, USA

\section{ARTICLE INFO \\ ABSTRACT}

\section{* Correspondence to:}

L. Lloyd Morgan

Environmental Health Trust, USA

e-mail: 1loyd.1.morgan@gmail.com

\section{Keywords:}

Adult

Children

Microwave radiation

Mobile phone

Specific absorption rate
History of exposure limits can be listed as follows; American National Standard Institute (ANSI) has identified that exposure limits were $10 \mathrm{~mW} / \mathrm{cm}^{2}$ averaged over 6 minutes in 1966 and 1974. Partial body irradiation must be included since it has been shown that some parts of the human body (e.g., eyes, testicles) may be harmed if exposed to incident radiation levels significantly in excess of the recommended levels. Today's standards allow partial body irradiation to be 20 times higher than whole body radiation. ANSI recognized the "the invariable presence of electrical hotspots in the irradiated body." In 1982. A threshold of behavioral disruption was established: $4 \mathrm{~W} / \mathrm{kg}$ " therefore a 10 -fold "safety factor" SARWB=0.4 W/kg. Behavioral disruption is irreversible: "The assumption is that reversible disruption during an acute exposure is tantamount to irreversible injury during chronic exposure". In 1991, Institute of Electrical and Electron Engineers (IEEE) mentioned two tier exposure level; partial body specific absorption rate (SAR) and whole body SAR are no longer identic. It was searched electrical workers and general public. Exposure of general public was calculated as SARWB=0 $08 \mathrm{~W} / \mathrm{kg}$; SARSP $=1.6$ $\mathrm{W} / \mathrm{kg}, 30$ minute average, also exposure of electrical workers calculated as SARWB $=0.4$ $\mathrm{W} / \mathrm{kg}$; SARSP=8 W/kg, 6 minute average. It has observed 5-fold lower SAR; 5-fold longer exposure between groups. Specific absorption rate is formulated as $\mathrm{SAR}=(\sigma / \mathrm{Q}) * \mathrm{E} 2$, where $\sigma$ is the conductivity of the tissue, $\varrho$ is the tissue density, and $\mathrm{E}$ is the electric field. However, $\mathrm{E}$ is a function of the electrical parameter permittivity, $\varepsilon$, "the resistance that is encountered when forming an electric field in a medium. Higher conductivity increases; higher tissue density decreases; higher permittivity decreases the SAR. However, children's absorption of microwave radiation is greater than adults. It was found that ICNIRP's $2 \mathrm{~W} / \mathrm{kg}, 10 \mathrm{~g}$ spatial peak SAR results in 2.3-3 times higher SAR than FCC's 1.6 $\mathrm{W} / \mathrm{kg}, 1 \mathrm{~g}$ spatial peak SAR. It was showed that $1-\mathrm{g}$ SAR of brain tissues of children is about two times higher than adults. At the same time another group of researchers asserted that hypocampus and hypothalamus receive 1.6-3.1 higher SAR in children compared to adults; children's bone marrow receive 10 times higher SAR than adults; children receive higher SAR to the eyes than adults; children's cerebellum receive $>2.5 \times$ higher SAR than adults. Greatly underestimating the SAR for typical mobile phone users, especially children. A superior computer simulation certification process has been approved by the Federal Communications Commission (FCC) but is not employed to certify cell phones. In the United States, the FCC determines maximum allowed exposures. Many countries, especially European Union members, use the "guidelines" of International Commission on Non-Ionizing Radiation Protection (ICNIRP), a non governmental agency. The SAR for a 10-year old is up to $153 \%$ higher than the SAR for the SAM model. When electrical properties are considered, a child's head's absorption can be over two times greater, and absorption of the skull's bone marrow can be ten times greater than adults. Therefore, a new certification process is needed that incorporates different modes of use, head sizes, and tissue properties.

J. Exp.Clin.Med., 2013; 30:270 